\title{
Wake Instabilities behind Discrete Roughness Elements in High Speed Boundary Layers
}

\author{
Meelan Choudhari, ${ }^{*}$ Fei Li ${ }^{*}$, Chau-Lyan Chang ${ }^{* *}$, Andrew Norris ${ }^{* * *}$, \\ NASA Langley Research Center, Hampton, VA 23681 \\ Jack Edwards ${ }^{\dagger}$, \\ North Carolina State University, Raleigh, NC 27695
}

\begin{abstract}
Computations are performed to study the flow past an isolated, spanwise symmetric roughness element in zero pressure gradient boundary layers at Mach 3.5 and 5.9, with an emphasis on roughness heights of less than 55 percent of the local boundary layer thickness. The Mach 5.9 cases include flow conditions that are relevant to both ground facility experiments and high altitude flight ("cold wall" case). Regardless of the Mach number, the mean flow distortion due to the roughness element is characterized by long-lived streamwise streaks in the roughness wake, which can support instability modes that did not exist in the absence of the roughness element. The higher Mach number cases reveal a variety of instability mode shapes with velocity fluctuations concentrated in different localized regions of high base flow shear. The high shear regions vary from the top of a mushroom shaped structure characterizing the centerline streak to regions that are concentrated on the sides of the mushroom. Unlike the Mach 3.5 case with nearly same values of scaled roughness height $\mathrm{k} / \boldsymbol{\delta}$ and roughness height $\mathrm{Reynolds}$ number $\mathbf{R e}_{\mathrm{kk}}$, the odd wake modes in both Mach 5.9 cases are significantly more unstable than the even modes of instability. Additional computations for a Mach 3.5 boundary layer indicate that the presence of a roughness element can also enhance the amplification of first mode instabilities incident from upstream. Interactions between multiple roughness elements aligned along the flow direction are also explored.
\end{abstract}

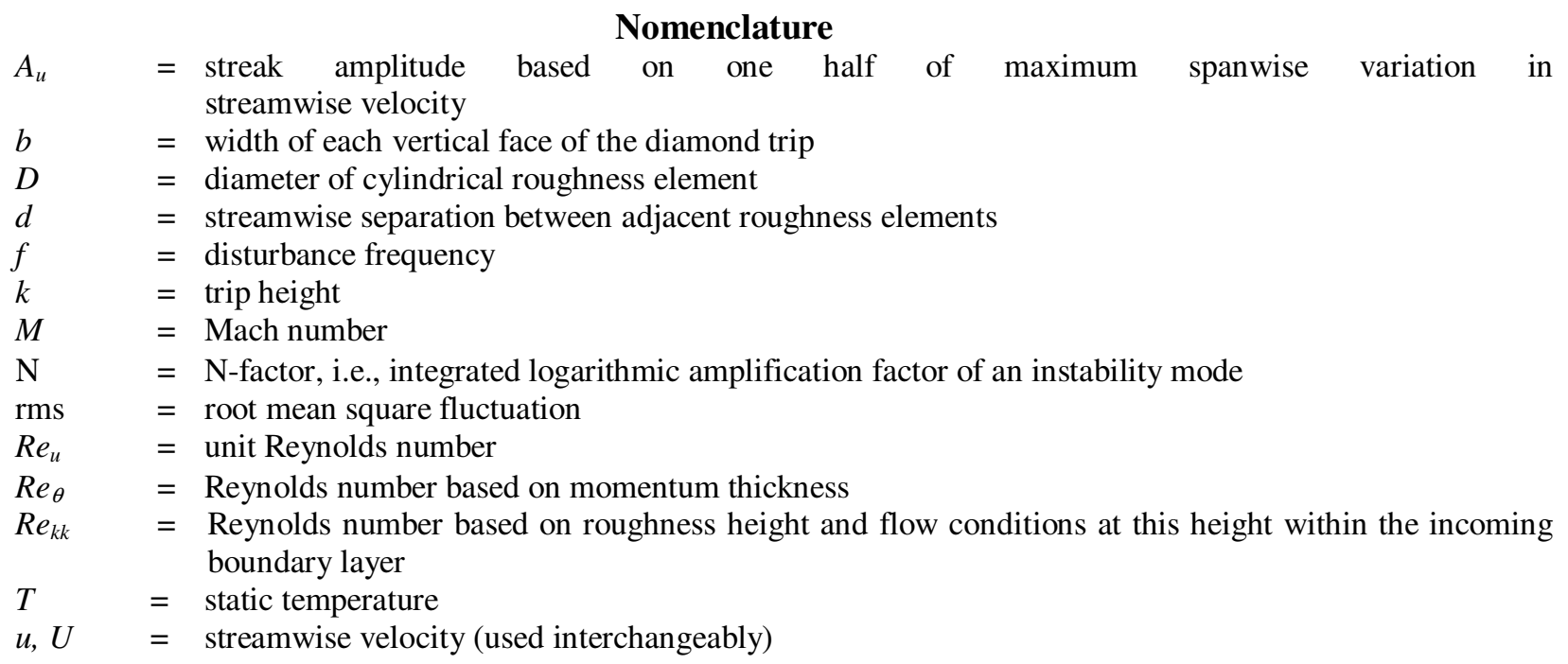

\footnotetext{
* Aerospace Technologist, Computational AeroSciences Branch, M.S. 128. Associate Fellow, AIAA. * Aerospace Technologist, Computational AeroSciences Branch, M.S. 128.

** Aerospace Technologist, Computational AeroSciences Branch, M.S. 128. Senior Member, AIAA.

*** Aerospace Technologist, Hypersonic Airbreathing and Propulsion Branch. Senior Member, AIAA.

$\dagger$ Professor, Department of Mechanical and Aerospace Engineering. Associate Fellow, AIAA.
} 


$\begin{array}{ll}w & =\text { frontal half width of trip } \\ (x, y, z) & =\text { streamwise, wall-normal, and spanwise coordina } \\ \delta & =\text { Boundary layer thickness based on } u / u_{\infty}=0.995 \\ \rho & =\text { density } \\ \Delta \mathrm{N} & =\text { Change in N-factor } \\ & \\ a d & =\text { adiabatic } \\ e & =\text { edge of unperturbed boundary layer } \\ k & =\text { trip height location } \\ r & =\text { location of roughness element } \\ t r & =\text { transition onset location } \\ w a l l & =\text { value at plate surface } \\ \infty & =\text { free-stream }\end{array}$

\section{Introduction}

Surface roughness is known to have a substantial impact on the aerothermodynamic loading in high Mach number flight, regardless of the state of the boundary layer along the vehicle surface. When the incoming boundary-layer flow is laminar, the presence of 3D surface roughness tends to accelerate the laminar-turbulent transition process, which can result in a dramatic increase in both drag and aerodynamic heating of the vehicle. In scramjet applications, artificial roughness elements are often employed for intentional tripping of the boundary layer on the forebody of the vehicle in order to prevent engine unstart and to minimize the flow non-uniformities at the entrance to the combustor inlet.

Empirical observations ${ }^{1,2}$ suggest that when the roughness height exceeds a critical value, the transition front begins to move upstream relative to that over a smooth surface in the same disturbance environment (Fig. 1). The rate of upstream movement slows down at sufficiently large heights and, for roughness heights larger than the so called effective trip height, the onset of transition appears to asymptote to a location ranging from somewhere just behind the roughness element to a finite distance downstream. Both the critical and effective values of the roughness height parameter are influenced by the shape of the roughness element and by the external disturbance environment. ${ }^{2}$

Despite the common occurence of surface roughness and its well-known impact on the vehicle design and operation for high-speed flight, the physical mechanisms underlying the transition process behind discrete roughness elements are not fully understood at this time. In principle, the roughness elements may lead to an earlier onset of transition via various physical mechanisms: (1) accelerated growth of existing instability modes, (2) new classes of instabilities in the trip modified flow, which may be convective modes within the intermediate to far wake behind the roughness element or absolute/global instabilities of the separated flow near the element, (vortex shedding, etc.) that may or may not be coupled to one another, (3) strong transient growth of boundary layer perturbations, and (4) enhanced receptivity to the existing disturbance environment. In practice, these mechanisms may operate on their own or, more likely, in concert with one another.

To help address some of the above mentioned mechanisms, the flow behind an array of ramp and diamond trips on a 1:3 scale model of the forebody of the Hyper-X vehicle was investigated in Ref. 3 . The flow in the wake of the trip array mounted on the first compression ramp of the forebody was shown to consist of prominent streamwise streaks, the signature of which had been observed in oil flow visualizations in the experiment. These streaks support multiple modes of convective instabilities and the growth of the instabilities was strong enough to cause transition onset before the end of the first ramp as measured during an earlier set of wind tunnel experiments. The concave streamline curvature associated with the ramp was noted to play a crucial role in amplification of the streaks near the compression corners and, hence, in sustaining the streak amplitudes over the length of the forebody (i.e., if the flow were to remain laminar for that long). The predicted role of convective instabilities in the wake is qualitatively consistent with the experimental observation ${ }^{2}$ that the disturbance environment has a significant effect on measured transition. 
Follow-on analysis ${ }^{4}$ confirmed that, due to the absence of concave streamline curvature over a flat plate boundary layer, the wake perturbation behind a roughness element in a Mach 3.5 boundary layer becomes weaker with increasing distance behind the roughness; yet the rate of decay is small enough to allow a significantly long region of wake instability that supports logarithmic amplification factors ( $\mathrm{N}$-factors) that are typically correlated with transition onset in a broad class of boundary layer flows. Variation of estimated transition onset location with respect to trip height was qualitatively consistent with the classical measurements. The numerical simulations ${ }^{4}$ also demonstrated that any general form of unsteadiness from the upstream flow can interact with the roughness element to excite the wake instability modes and, hence, no additional ingredients are required for the receptivity process associated with these modes.

The flow behind an isolated roughness element was further investigated by Chang et al., ${ }^{5}$ who addressed the potential onset of spontaneous unsteadiness behind a roughness element of height that is close to the boundary layer thickness. It was shown that the wake flow spontaneously became unsteady at sufficiently large roughness heights, exhibiting a complex interplay between the unsteadiness in the separation region immediately upstream of the roughness element and the disturbance motion farther downstream within the wake. The latter interplay indicated the possibility of coupling between the local instabilities of the wake flow and absolute instabilities near the roughness element. Strong tonal perturbations amplifying within the near-wake region were also detected via measurements of surface pressure fluctuations in quiet flow experiments ${ }^{6}$ at $\mathrm{M} \approx 6$ and recent numerical simulations of that experiment. ${ }^{7}$ Subsonic boundary layers with roughness elements are also known to support absolute instabilities, ${ }^{8-10}$ but those appear to be associated with the separation region immediately behind the roughness element rather than ahead of it. Roughness induced transition is currently an active area of research and the reader is referred to Refs. 11-21 for additional findings in this area.

The main goal of the present paper is to extend the previous computations of roughness wake instabilities from supersonic to hypersonic Mach numbers. It is important to examine the effects of the increase in Mach number since, even in the absence of the roughness element, the dominant instability mechanism changes from first mode waves at supersonic Mach numbers to second mode instabilities at hypersonic speeds. Even at hypersonic flight speeds, the Mach number at the edge of the boundary layer can vary over a broad range including hypersonic, supersonic, and even subsonic Mach numbers depending on the geometric configuration and angle of attack. The higher Mach number configurations examined in Section 2 mimic the roughness geometry and roughness height Reynolds number from the earlier Mach 3.5 computations, ${ }^{4}$ but for flow conditions that are representative of a Mach 6 wind tunnel experiment and Mach 6 high altitude flight, respectively. Local growth rate characteristics at selected stations within the roughness element wake are examined first, followed by the streamwise variation of integrated amplification factors with and without the roughness element. To help understand the roughness effects at postcritical heights (whereby the roughness height is just large enough to begin having an impact on the transition onset location), the interaction between incoming instability waves and a roughness element of suitably small height (such that the streak instability is weak and cannot lead to transition) is examined for the Mach 3.5 configuration in Section 3. Finally, the wake flow behind multiple roughness elements stacked along the streamwise direction at relatively small spacing is investigated in Section 4. Concluding remarks are presented in Section 5.

\section{Streak Instabilities}

\section{A. Flow Configurations}

The unperturbed boundary layer flow from the Mach 3.5 configuration relevant to the Supersonic Low Disturbance Tunnel (SLDT) at NASA Langley only supports first mode instabilities, whereas the dominant instabilities in boundary-layer flows at higher Mach numbers are of the second mode (or Mack mode) type. Given these differences, it is important to examine whether the wake instability mechanisms identified in our previous analyses ${ }^{3,4}$ are also operative at the higher edge Mach numbers relevant to hypersonic air-breathing propulsion. To that end, computations have been carried out for a nominally Mach 6 boundary layer for two separate flow conditions that are representative of wind tunnel and flight conditions, respectively. For brevity, these cases are denoted as M6T and M6F, respectively. The wind tunnel condition for case M6T corresponds to a unit Reynolds number of $\operatorname{Re}_{u}=$ $9.84 \times 10^{6} / \mathrm{m}$, the free-stream temperature $T_{\infty}=54.17 \mathrm{~K}$, and the surface temperature normalized with respect to adiabatic surface temperature corresponds to $T_{\text {wall }} T_{a d}=0.9$. The flight condition (M6F) corresponds to a unit Reynolds number of $R e_{u}=2.07 \times 10^{6} / \mathrm{m}$ and free-stream and surface temperature corresponding to $T_{\infty}=226.94 \mathrm{~K}$ and $T_{\text {wall }} / T_{a d}=0.25$, respectively. In both cases, the dimensions of the diamond trip are scaled by a uniform scaling 
factor with respect to the Mach 3.5 configuration from Ref 4 (denoted herein as case M3.5T) and the streamwise location is adjusted to achieve (partial) dynamic similarity by keeping the non-dimensional parameters $\operatorname{Re}_{k k}$ (Reynolds number based on roughness height and flow conditions at that height) $\approx 547$ to $575, k / \delta$ (roughness height scaled by boundary layer thickness) $\approx 0.55$, and $b / k$ (roughness width to height ratio) $\approx 3.33$ approximately the same as those in the supersonic Mach 3.5 case. The roughness dimensions for the M3.5T trip correspond to $k=0.381 \mathrm{~mm}$ and $b=1.27 \mathrm{~mm}$ and both of them are scaled up for the M6T and M6F cases by factors of 5.43 and 4.42, respectively. A schematic of the roughness element geometry is presented in Fig. 2, and Table I provides a summary of the flow conditions for each of the three cases of interest.

The mean flow in the presence of the roughness element is computed by solving the compressible Navier-Stokes equations using the same computational methodology as that used for the M3.5T case ${ }^{4}$ and, in fact, the computational mesh is also obtained via a suitable translation and uniform scaling of the mesh used in Ref 4 . The mesh involves 1601 points in the streamwise direction, 289 points normal to the plate, and 177 points along the spanwise direction, although spanwise symmetry is imposed to reduce the spanwise grid count to 89 . The basic state computed in this manner is used as input for the linear stability analysis of the roughness wake.

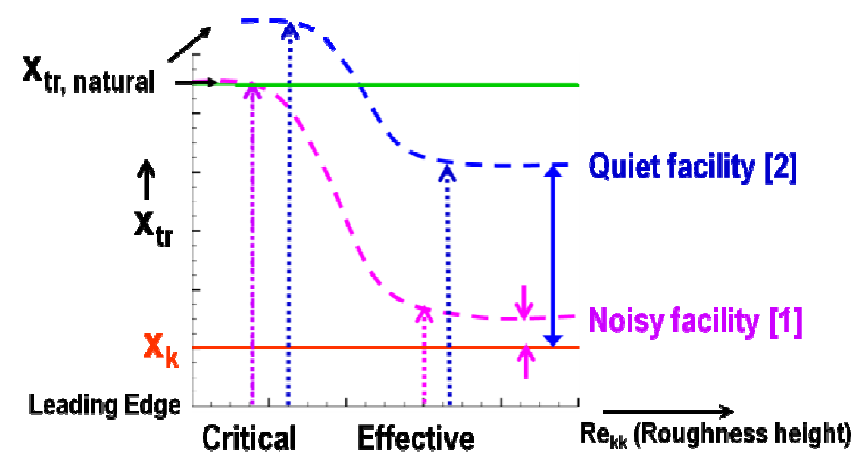

Figure 1. Schematic of variation in transition location with roughness height parameter.

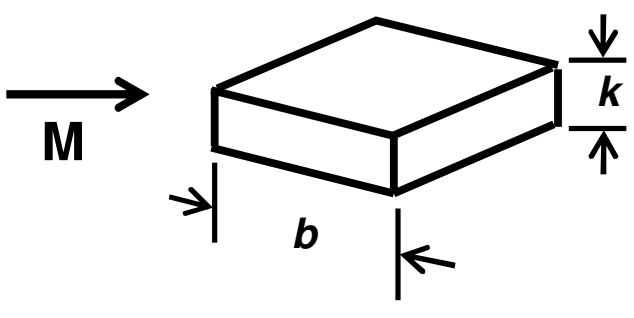

Figure 2. Schematic of diamond trip. The face width $b$ is related to frontal half width $w$ via $w=b \cos (\pi / 4)$.

Table I: Summary of Flow Configurations

\begin{tabular}{|c|c|c|c|c|c|c|c|}
\hline case & $M_{\infty}$ & $\operatorname{Re}_{u}\left(m^{-1}\right)$ & $T_{\infty}(K)$ & $x_{r}(m)$ & $T_{\text {wall }} / T_{a d}$ & $\operatorname{Re}_{k k}$ & $\operatorname{Re}_{\theta} M_{e} k / \delta$ \\
\hline M3.5T & 3.5 & $9.89 \times 10^{6}$ & 87.0 & 0.0411 & 1.00 & 547 & 66 \\
\hline M6T & 5.91 & $9.84 \times 10^{6}$ & 54.2 & 0.3853 & 0.25 & 575 & 119 \\
\hline M6F & 5.91 & $2.07 \times 10^{6}$ & 226.9 & 0.1985 & 0.90 & 567 & 35 \\
\hline
\end{tabular}

\section{B. Roughness Height Parameters}

Selected characteristics of the unperturbed, self-similar boundary layer flow for the cases of interest are examined first. The roughness height parameters $R e_{k k}$ and $R e_{\theta} M_{e} k / \delta$ are commonly used to predict transition due to surface roughness. ${ }^{22,23}$ Profiles of $R e_{k k}$ (accompanied by static temperature profiles) as functions of the roughness height have been plotted in Fig. 3(a), whereas the corresponding profiles of unperturbed, mean flow Mach number are shown in Fig. 3(b). Fig. 3(a) shows that, in spite of the differences in free-stream Mach number, the profiles of both $R e_{k k}$ and the normalized temperature are nearly the same for the M3.5T and M6F cases, with the exception of differences between the near-wall temperature profiles due to differences in the thermal boundary conditions (adiabatic boundary condition for M3.5T and cold wall for M6F). On the other hand, the $R e_{k k}$ profile for the M6T case exhibits a steep increase at larger roughness heights $(k / \delta>0.75)$ and, hence, rapidly deviates from the other two cases. For the M3.5T and M6F cases, the increase in $R e_{k k}$ with $k / \delta$ is approximately quadratic up to $k / \delta \approx 0.4$. 


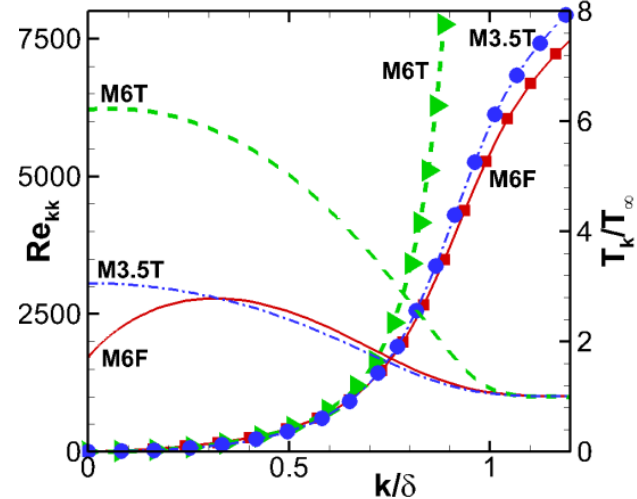

(a) Roughness height Reynolds number (lines with symbols) and static temperature (lines without symbols)

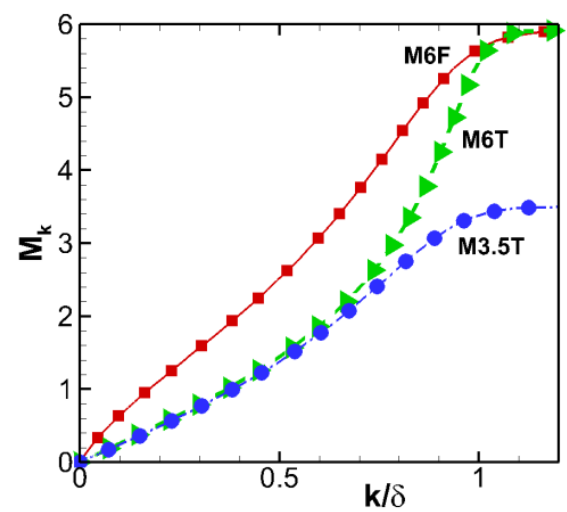

(b) Mach number

Figure 3. Boundary layer profiles.

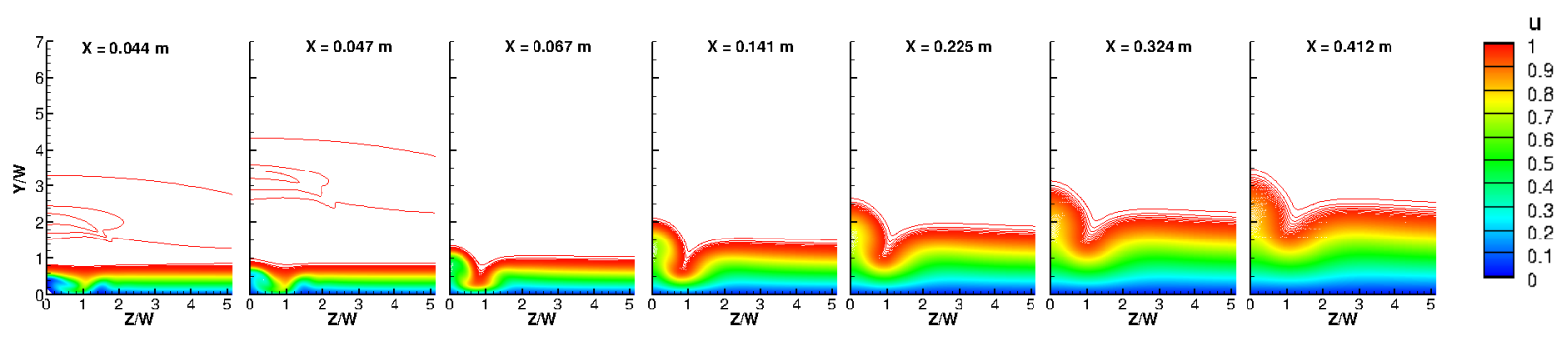

(a) M3.5T
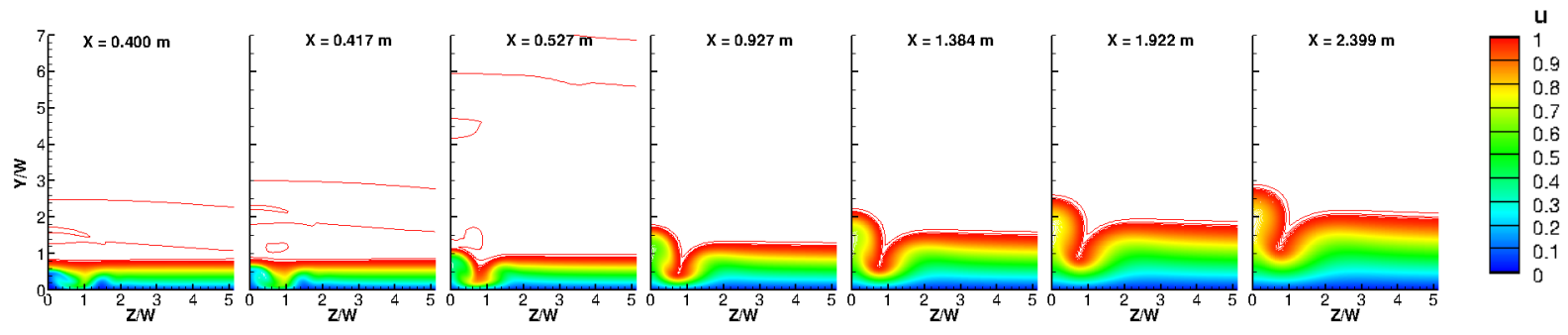

(b) M6T
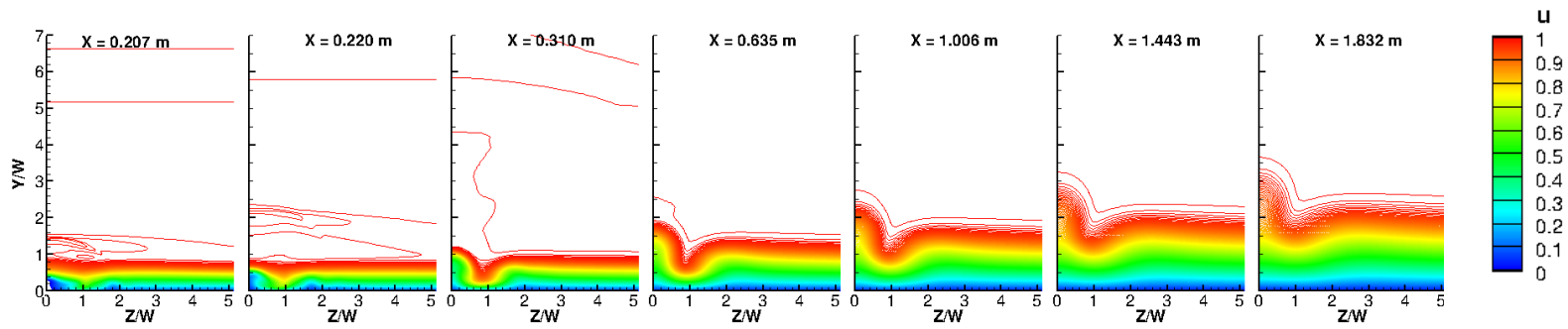

(c) $\mathrm{M6F}$

Figure 4. Streamwise velocity contours in roughness wake for flow configurations from Table I. Due to spanwise symmetry of the flow field, only the right half of the wake region is shown. Both abscissa and ordinate are normalized by the frontal half width $w=b \cos (\pi / 4)$. Selected stations correspond to approximately same values of the non-dimensional parameter $\left(x-x_{r}\right) / k \approx 7,15,68,262,482,742,973$. 
The variation in roughness height Mach number for the M6T case is close to that in the M3.5T case for $k / \delta<0.7$. In contrast, the Mach number profile for the M6F case has a larger slope at $k / \delta=0$ and, hence, does not approach the M6T profile until the very edge of the boundary layer. At the roughness height of interest in this paper $(k / \delta \approx 0.55)$, the local Mach number is moderately supersonic $\left(M_{k} \approx 1.5\right)$ for M3.5T and M6T cases, but somewhat larger $\left(M_{k} \approx\right.$ $2.5)$ in the $\mathrm{M} 6 \mathrm{~F}$ case. Unlike the $R e_{k k}$ variation, the roughness height parameter $R_{\theta}{ }_{d} M_{e} k / \delta$ from Berry et al. ${ }^{12}$ varies linearly with $k / \delta$ and, hence, does not display the sharp increase in $R e_{k k}$ at large roughness height for case M6T. It will be useful to design future experiments to focus on such differences to better understand the limitations of the empirical criteria for effective tripping that are based on easily available mean boundary layer parameters.

\section{Mean Wake Flow}

The modified mean flow in the presence of the roughness element was computed using an immersed boundary method (IBM) as described in Ref 4. We note that the possibility of self-sustained unsteadiness in the immediate vicinity of the roughness element cannot be ruled out entirely. ${ }^{5}$ However, the main focus herein is to compare the stability characteristics in the mean wake flow and, since the L2 norms of the residual levels had dropped to $\mathrm{O}\left(10^{-11}\right)$ or less when they leveled off, it was assumed that the wake flow computed via local time stepping would provide a meaningful basic state for the analysis of wake instabilities. Future computations are planned to verify this assumption as well as to confirm the accuracy of the IBM in the Mach 6 cases.

As described in Ref 4, the presence of the roughness element leads to a small region of flow reversal in the vicinity of the trip. At the intermediate roughness heights under consideration, the intermediate to far wake flow is dominated by a strong upwelling near the centerline where the roughness induced perturbation causes the boundary layer thickness to be substantially larger in comparison to the unperturbed flow. The centerline streak characterized by mushroom shaped contours of streamwise velocity is surrounded by a pair of streamwise streaks with reduced boundary layer thickness. The streaky structures remain prominent for large distances behind the roughness element (Figs. 4(a)-4(c)). Despite the differences in Mach number and thermal boundary conditions, the axial velocity contours at streamwise locations corresponding to same values of $\left(x-x_{r}\right) / k$ are similar in all three cases as seen from Figs. 4(a)-4(c). The high-speed streaks corresponding to reduced boundary layer thickness appear to be the strongest in the case M6T and weakest in the case M6F.

The roughness element shape examined in Figs. 4(a)-4(c) has a planform size larger than the local thickness of the unperturbed boundary layer $(2 w / \delta \approx 2.6)$. In contrast, the diameter $D$ of the cylindrical roughness element employed in the Mach 6 quiet tunnel experiments at Purdue University ${ }^{6}$ was significantly smaller than the estimated boundary layer thickness $(D / \delta \approx 0.7)$. To help assess the effects of planform width on the mean wake structure, preliminary computations of the wake flow behind a cylindrical roughness element of height $2.54 \mathrm{~mm}(\mathrm{k} / \delta \approx 0.3)$ at the conditions of the Purdue experiment (stagnation pressure of $6.067 \times 10^{6} \mathrm{~Pa}$, i.e., $88 \mathrm{psi}$, and stagnation temperature of $424 \mathrm{deg} \mathrm{K}$ ) are shown in Fig. 5. The qualitative features of the wake contours are analogous to those in Fig. 4 for a larger planform size, with a progressive roll-up of slower moving fluid in the vicinity of the centerline as the wake flow transition from near wake to farther downstream. Consistent with the shorter spanwise scale for the cylindrical element, the spanwise gradients of the wake flow appear to be stronger in Fig. 5 in comparison to those shown in Fig. 4 for the wider diamond trip. Further computations are necessary to determine what effect, if any, the narrower spanwise width of the wake has on the instability characteristics. 

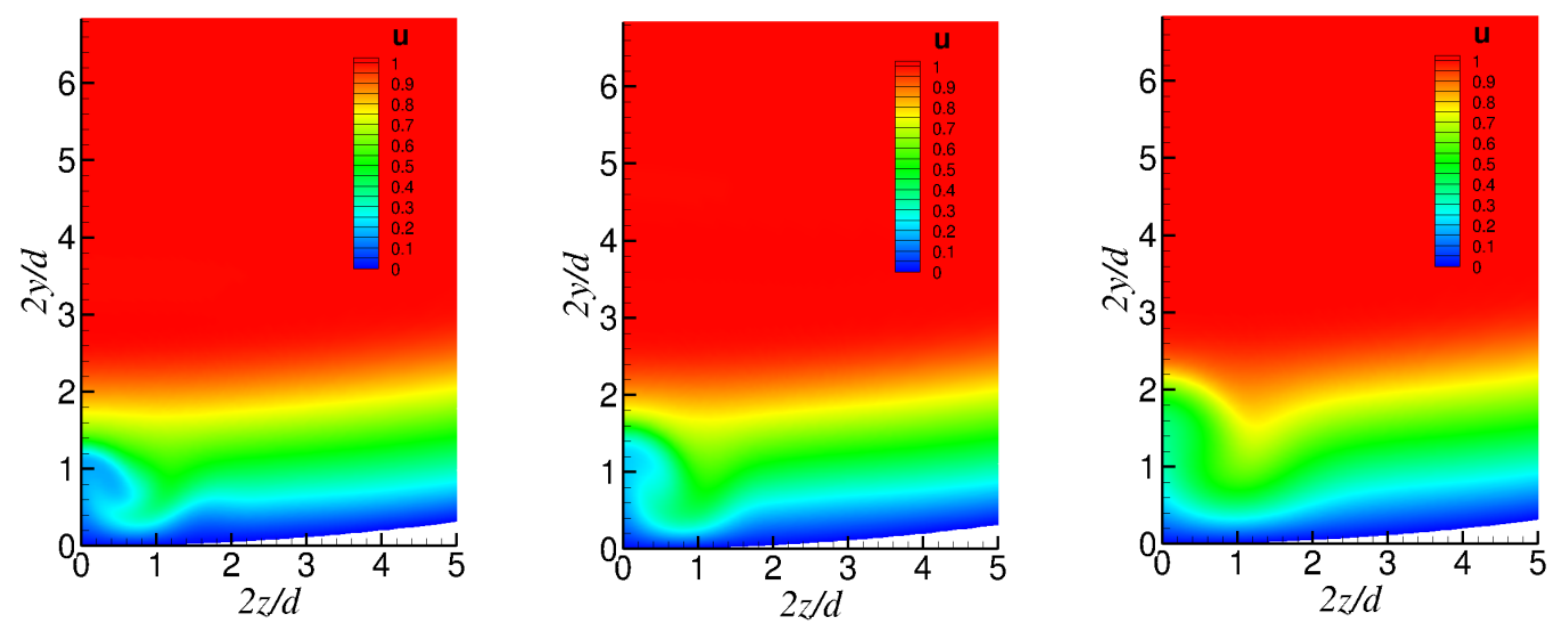

Figure 5. Streamwise velocity contours behind a cylindrical roughness element with $k=2.54 \mathrm{~mm}$ in the experiment by Wheaton et al. ${ }^{6}$ Selected stations correspond to $\left(x-x_{r}\right) / k \approx 7,15,68$.

\section{Local Instability Characteristics}

Next, the stability of the wake behind the diamond roughness element is studied for cases M6T (Fig. 4(b)) and M6F (Fig. 4(c)). Since the spanwise and wall normal length scales of the boundary layer streaks within the wake are comparable, the modified boundary layer flow has a strongly inhomogeneous character in both $y$ and $z$ directions. Therefore, its stability characteristics are more appropriately studied by solving an eigenvalue problem based on two-dimensional partial differential equations (PDEs), ${ }^{24,25}$ rather than using the conventional linear stability analysis, which is based on the assumption of basic state inhomogeneity in a single spatial coordinate (viz., the surface normal direction). The spatial stability of the computed streaks was examined at three selected streamwise locations in the roughness wake. The spatial grid consisted of 131 appropriately clustered points in the surface normal direction and 67 points in the spanwise direction.

As discussed in Ref. 4, the wake flow in the M3.5T case supports both even (symmetric or varicose) and odd (antisymmetric or sinuous) modes of instability. To enable separate computation of these two modes, the spanwise domain was limited to positive values of the $z$ coordinate. The fluctuations associated with both even and odd modes are concentrated over the narrow spanwise extent of the streaks, which is significantly smaller in comparison to the spanwise wavelength of the dominant first mode instability of the unperturbed boundary layer flow. In the limited cases where the mode shapes were examined, fluctuations associated with the dominant even mode were concentrated near the top of the mushroom-like structure associated with secondary flow upwelling, i.e., in the region of increased wall-normal shear $(d u / d y)$. The dominant odd modes were concentrated on either side of the mushroom structure, where the spanwise shear $(d u / d z)$ is large. Analogous stability predictions for the Mach 6 cases are presented below, and more attention has been paid to the mode shapes at each of the three selected stations examined herein.

\section{Case M6T}

The spatial growth rates of first and second mode instabilities of the boundary layer flow in the absence of any roughness element are plotted in Fig. 6 for three selected stations in the case M6T. The growth rates of dominant even and odd mode instabilities at the same stations but in the presence of the diamond roughness from Table I are plotted in Figs. 7(a) and 7(b), respectively. Figure 6 shows that as the (unperturbed) boundary layer grows in the downstream direction, the frequency of the locally most unstable second mode disturbance decreases from approximately $80 \mathrm{kHz}$ at the first station to approximately $50 \mathrm{kHz}$ at the third station. The most unstable first mode disturbances correspond to lower frequencies, in the range of approximately $10 \mathrm{kHz}$ to $30 \mathrm{kHz}$.

Unlike the Mach 3.5 case from Ref. 4, the frequency range of unstable disturbances in the roughness wake for case M6T (Fig. 7) has a greater overlap with the frequencies of unstable disturbances corresponding to the unperturbed boundary layer (Fig. 6). Comparing the left and middle plots from Fig. 7(a), one observes that the peak growth rates of even modes at the first two stations $\left(x-x_{r}\right) / k \approx 47$ and 92 , respectively, are close to each other, with the peak 
growth rate at the second station being the slightly larger of the two. The peak growth at the farthest downstream wake station $\left(\left(x-x_{r}\right) / k \approx 399\right.$, the rightmost plot in Fig 7(a)) is smaller in comparison with the two upstream stations, but by less than 25 percent. For both dominant even modes corresponding to the peak growth rate at the first station, the highest streamwise velocity fluctuations are concentrated on either side of the mushroom structure in the base flow velocity contours, where the spanwise shear $(d u / d z)$ associated with the basic state is large. For brevity, these modes are referred to as $\mathrm{S}$ modes (short form for the side region). The PDE based instability analysis also revealed an additional unstable mode at higher frequencies, whose peak fluctuations are concentrated outside the main mushroom, i.e., within the region of relatively unperturbed mean flow in the exterior region. This mode is referred to as the outer $(\mathrm{O})$ mode or, equivalently, a boundary layer mode as opposed to the $\mathrm{S}$ modes, which are concentrated in the interior and, therefore, may be grouped under the general classification of "wake" modes. The complete physical significance of the outer mode is not clear at this time, beyond the obvious fact that it must be related to the second mode instability of the unperturbed boundary layer flow. The growth rate predictions for this mode are independent of grid resolution, but do vary somewhat as the spanwise extent of the computational domain is changed. Further work on establishing its significance is currently under way. All of the remaining PDE based instability predictions in this paper are confined to the wake modes.

At the most upstream station in Fig. 7(a), there is a clear separation between the frequency range of the wake modes and the outer mode, respectively. At the two downstream locations (corresponding to the middle and rightmost plots in Fig. 7(a)), the frequency range of the wake modes becomes broader and extends to higher frequencies than those at the first station. Simultaneously, the frequencies of the outer mode decrease with downstream distance (not shown), presumably as a result of the thickening of the unperturbed boundary layer. The above trends lead to an overlap between the frequencies of the wake modes and outer mode and, hence, one encounters hybrid modes with significant fluctuations in both the inner (wake) region and the outer region at the latter two stations. Again, consistent with our focus on the wake modes in the $2 \mathrm{D}$ eigenvalue analysis, any modes with significant fluctuations in the outer region are omitted from further discussion. Integrating the growth rates along the wake also verified that the $\mathrm{N}$-factors associated with the wake modes increased much more rapidly with distance than the $\mathrm{N}$-factors associated with second mode amplification in the unperturbed boundary layer, confirming the possibility of roughness induced bypass transition due to the wake modes (i.e., streak instabilities).

The two downstream locations in Fig. 7(a) also exhibit a broader variety of even modes with comparable values of peak growth rates. Similar to station 1, the peak growth rate at station 2 corresponds to the $\mathrm{S}$ mode (Fig. 8(a)), but there are additional modes with dual regions of concentration. The SI mode has a primary peak on the side and a secondary peak on the inner side of the wake region beyond the roughness edge (Fig. 8(b)), whereas mode CS (Center-Side) mode (not shown in Fig. 7(a)) has a primary peak near the centerline along the top of the mushroom and a secondary one on the side of the mushroom (Fig. 8(c)). Although not shown, the mode shapes at the farthest downstream station indicate more than two regions of concentration as indicated by the mode label CSI at that station.
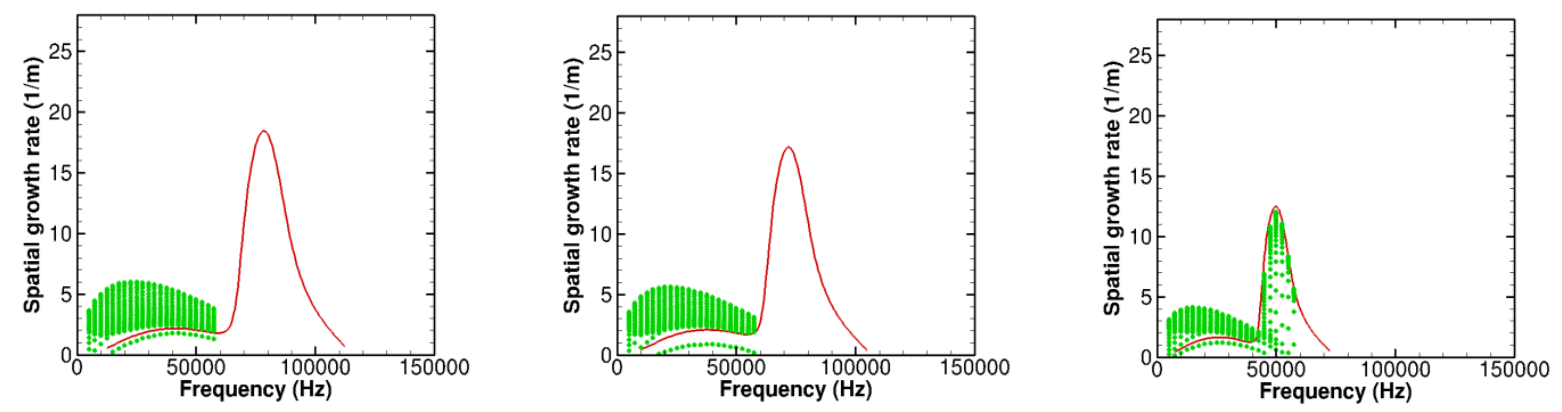

Figure 6. Variation of local growth rate with frequency at selected streamwise stations for first and second mode instabilities of unperturbed boundary layer in case M6T (from left to right: $(x$ $\left.x_{r}\right) / k \approx 47,92,399$, respectively; red curve: $2 \mathrm{D}$ modes, green symbols: oblique modes of various spanwise wavelengths). 

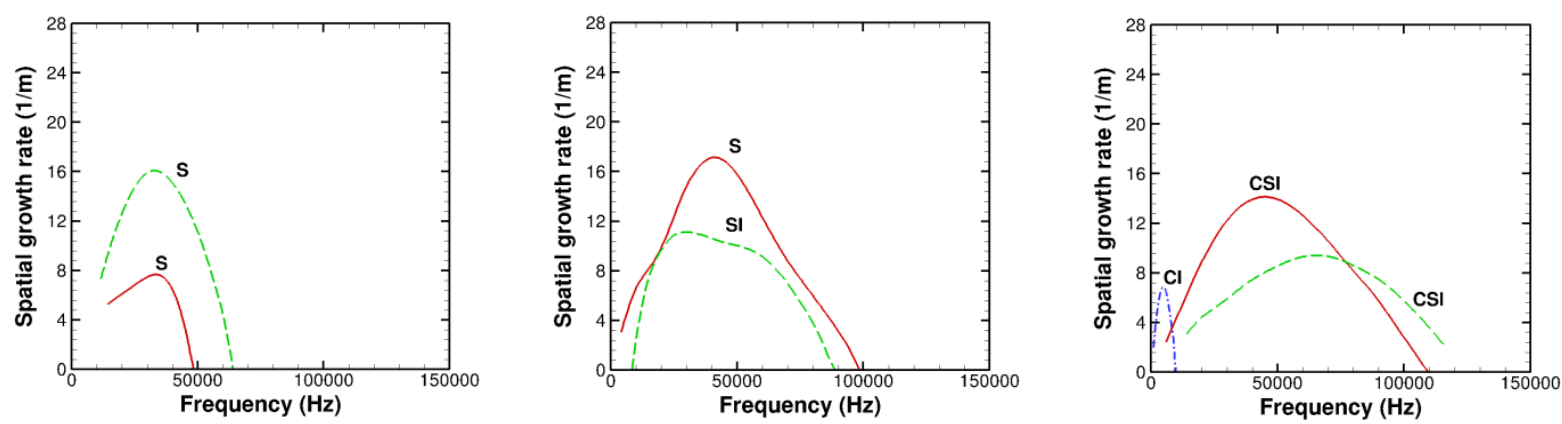

(a) Even (i.e., symmetric) modes.
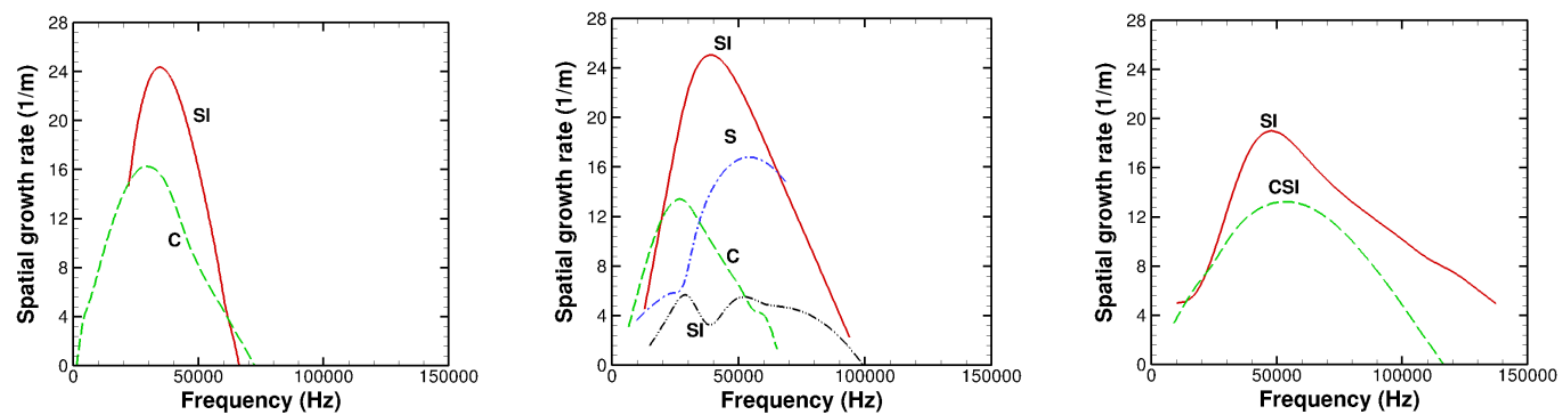

(b) Odd (i.e., antisymmetric) modes.

Figure 7. Variation of local growth rate with frequency at selected streamwise stations for case M6T (from left to right, stations 1 to 3 correspond to $\left(x-x_{r}\right) / k \approx 47,92,399$, respectively). The letters identifying each curve correspond to the region(s) of peak streamwise velocity fluctuation at the frequency corresponding to the peak growth rate for that curve as described in Figs. 8 and 9 and the corresponding text. Only selected families of unstable modes are shown, and curves of same color between different stations indicate topological continuations of each other.

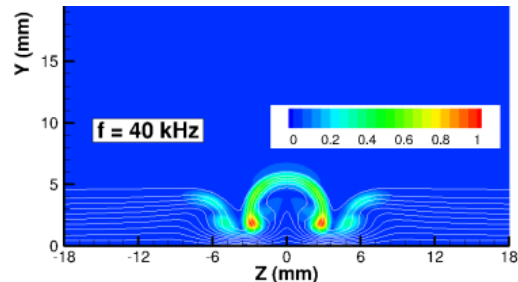

(a) Mode $\mathrm{S}$

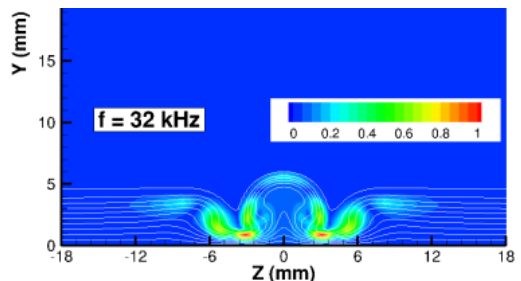

(b) Mode SI

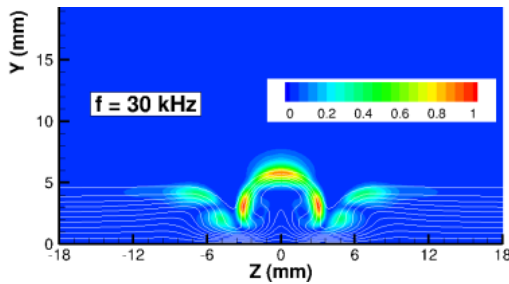

(c) Mode CS

Figure 8. Normalized mode shapes for streamwise velocity fluctuation associated with even modes corresponding to peak growth frequency at $x=0.575 \mathrm{~m}\left(\left(x-x_{r}\right) / k \approx 92\right)$ in case M6T. White lines in each plot indicate the contours of basic state $u$-velocity distribution.

Overall, the peak growth rates associated with the odd modes in case M6T (Fig. 7(b)) are larger than those corresponding to the even modes (Fig. 7(a)). At each of the three locations shown in Fig. 7(b), the peak growth rate of the odd wake modes is nearly 50 percent larger than the corresponding value for the even wake modes. At all three locations, the highest local growth rate corresponds to an odd mode disturbance that is concentrated in two adjacent areas of high spanwise shear, namely the side of the mushroom structure and the inner edge of the region exterior to the mushroom. (See Fig. 9(a) for the mode shape for odd SI mode at station 2.) The peak growth rate of the second odd mode at the farthest upstream station (leftmost plot in Fig. 7(b)) is nearly equal to the overall maximum of the growth rate of the even modes at the same station (leftmost plot in Fig. 7(a)). The streamwise velocity fluctuations associated with odd modes corresponding to the second and third highest peak growth rates at station 2 are concentrated near the side of the mushroom structure (mode S, Fig. 9(b)) and just outside the centerline 
(near-centerline mode C from Fig. 9(c)), respectively. Of course, by virtue of the odd parity, the streamwise fluctuations are identically zero at the center (i.e., the top) of the mushroom and the peak velocity fluctuations for mode $\mathrm{C}$ occur between the centerline and the region of high spanwise gradients on the side of the mushroom.

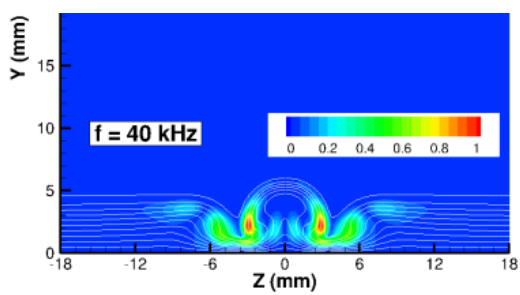

(a) Mode SI

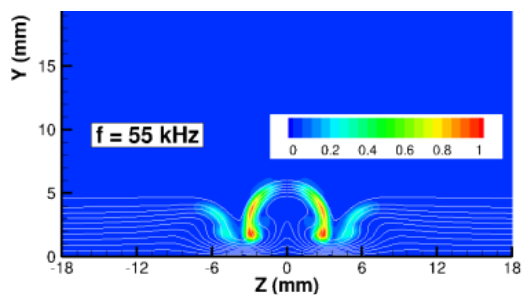

(b) Mode $\mathbf{S}$

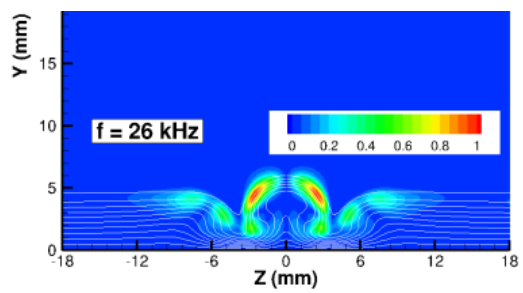

(c) Mode C

Figure 9. Normalized mode shapes for streamwise velocity fluctuation associated with odd modes corresponding to peak growth frequency at $x=0.575 \mathrm{~m}\left(\left(x-x_{r}\right) / k \approx 92\right)$ in case M6T.

Although not shown in this paper, the instability characteristics of the centerline flow were examined using the simpler classical stability analysis based on ordinary differential equations (rather than 2D PDEs based on the crosssection of the streamwise elongated wake structure). The growth rate characteristics based on the classical analysis were significantly different from those discussed above, both in terms of the range of unstable frequencies at a given station and the associated mode shapes and growth rates, indicating the need to use the more advanced PDE based stability analysis in general. Furthermore, it should be noted that the odd modes of instability in the wake have zero streamwise velocity fluctuations along the centerline and, hence, cannot be predicted using classical stability analysis of the centerline flow.
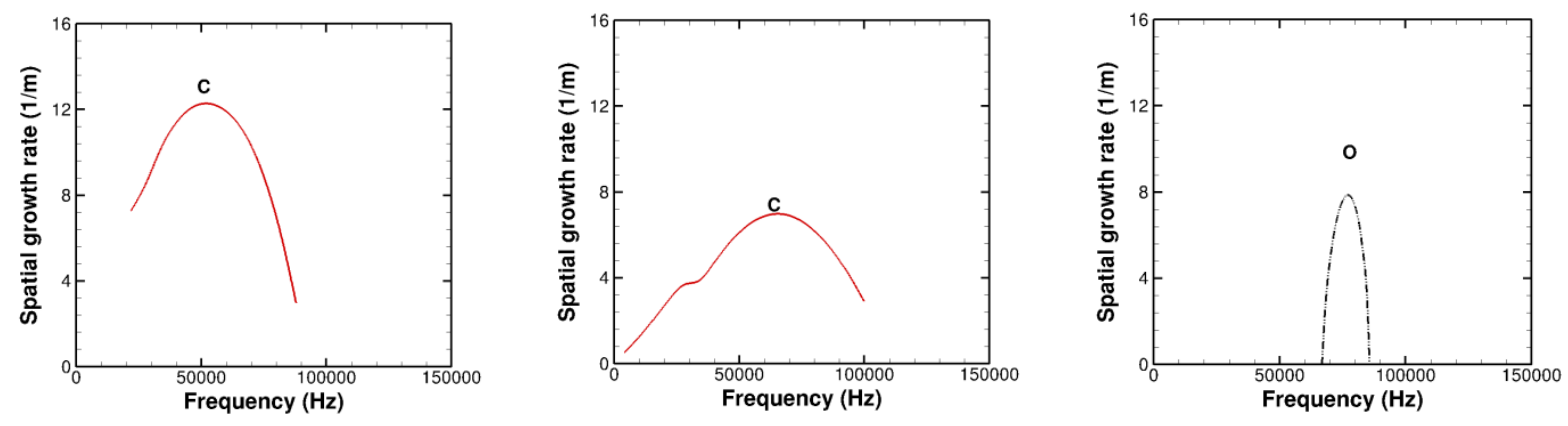

(a) Even (symmetric) modes.
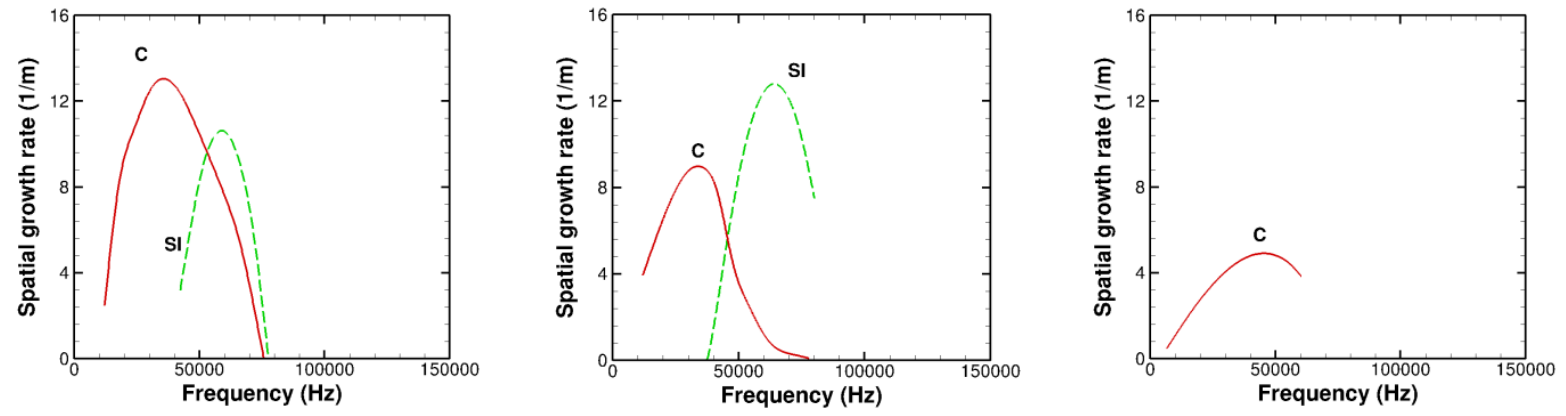

(b) Odd (antisymmetric) modes.

Figure 10. Variation of local growth rate with frequency at selected streamwise stations for case M6F. The letters identifying each curve correspond to the region(s) of peak streamwise velocity fluctuations in the mode shape at the frequency corresponding to peak growth rate for that curve as described in Figs. 6 and 7 and the corresponding text. Only selected families of unstable modes are shown. 


\section{Case M6F}

The growth rate characteristics for the M6F case are examined next. Besides having lower overall magnitudes, the growth rates for the M6F case (Fig. 10) indicate some significant differences from those in Fig. 7 for the M6T case. First, the modal complexity is reduced in the M6F case, with fewer families of unstable wake modes with strong local amplification at the selected stations. Specifically, there is a single wake mode of the even type and it undergoes amplification over a relatively short distance, becoming stable at the farthest downstream station. Similar to the even mode disturbances encountered in the M3.5T case, ${ }^{4}$ the streamwise velocity fluctuations associated with this even wake mode (mode C) are concentrated near the top of the mushroom structure, i.e., in the region of large wall-normal gradients in the basic state. At station 3, only the outer mode is unstable and it has been included here for illustration. There is one family of odd modes that has peak fluctuations along the top of the mushroom structure, somewhat away from the centerline (mode C in Fig. 10(b)). The peak growth rate associated with this C family of odd wake modes decreases with distance from the roughness element, but these modes continue to amplify over the range of streamwise locations covered in Fig. 10(b). The highest fluctuations associated with the second family of odd wake modes (mode SI) are concentrated in both regions of high spanwise gradients as discussed in the context of odd SI modes in the M6T case (Fig. 9(a)). The peak growth for the SI family increases from station 1 to station 2, but these disturbances appear to become stable somewhere between the second and third stations. The "C" family of odd disturbances is expected to achieve higher overall amplification factors because of the longer region of amplification and, hence, is likely to play a more significant role in accelerating the onset of transition relative to that due to second mode amplification in the unperturbed boundary layer.

\section{N-Factor Evolution}

The results presented so far focused on the local growth rate characteristics at selected locations within the wake. Since the significance of the various instability modes during the overall transition process depends on the integrated amplification factors (i.e., $\mathrm{N}$-factors), the $\mathrm{N}$-factor evolution for the unperturbed boundary layer flows is shown in Figs. 11(a)-(b) (case M6T) and Fig. 11(c) (case M6F), respectively. Figure 12 shows the streamwise evolution of the $\mathrm{N}$-factor for disturbance modes of selected frequencies in the presence of the roughness element. The $\mathrm{N}$-factor computation for the streak instabilities in the wake is initiated just behind the roughness element and the selected frequencies fall within the range of the most amplified instability modes. Figure 11(a) shows that the N-factors of the oblique first mode disturbances in case M6T are comparable to the N-factors for second mode disturbances (Fig. 11(b)), but the overall amplification of the 2D second mode disturbances is larger in comparison to the oblique first mode disturbances. Second mode disturbances with a frequency of approximately $50 \mathrm{kHz}$ achieve an $\mathrm{N}$-factor of 9 near $x=1.7 \mathrm{~m}$. On the other hand, the unperturbed boundary layer flow at conditions M6F is relatively more stable (mainly because of the lower unit Reynolds number) and, hence, the $\mathrm{N}$-factors are relatively lower over a comparable distance along the surface.

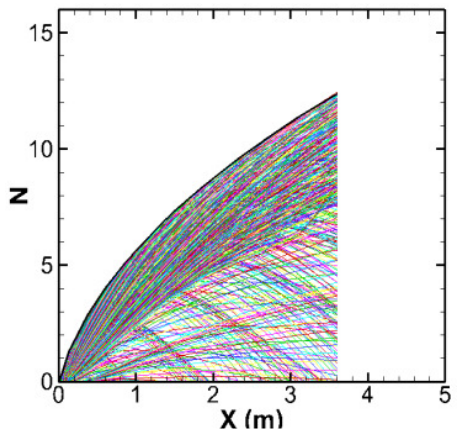

(a) Case M6T (oblique modes with wave angle greater than 30 degrees)

Figure 11. Streamwise evolution of integrated logarithmic amplification ratios (i.e., $\mathrm{N}$ factors) for unperturbed boundary layer flows.

The results in Fig. 12 confirm the destabilizing influence of the diamond roughness. Specifically, both even and odd modes of wake instability achieve higher $\mathrm{N}$-factors much earlier than the instability modes of the unperturbed boundary layer. Specifically, the even modes achieve $\mathrm{N}=9$ at $x \approx 1 \mathrm{~m}$ whereas the odd modes achieve the same $\mathrm{N}$ factor near $x \approx 0.83 \mathrm{~m}$, i.e., at less than half the distance required for the second mode instabilities of the unperturbed 
boundary layer. The wake instabilities are analogously stronger in the M6F case, even though the peak N-factor does not reach $\mathrm{N}=9$ within the length of the computational domain.

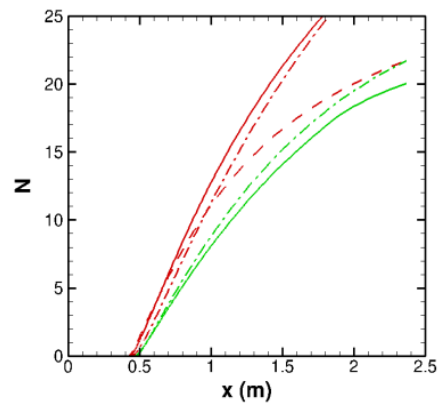

(a) Case M6T (odd modes)

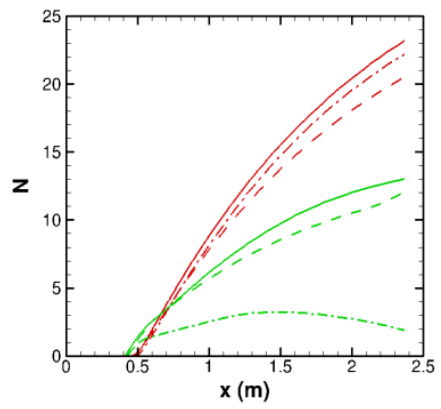

(b) Case M6T (even modes)

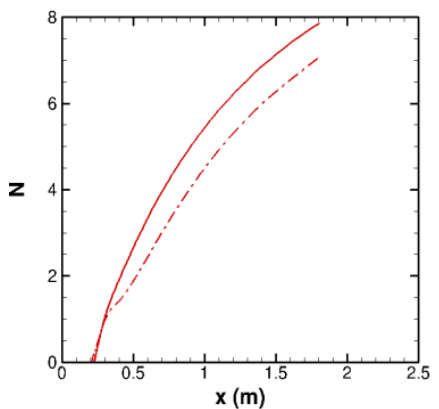

(c) Case M6F (odd modes)

Figure 12. Streamwise evolution of integrated logarithmic amplification ratios (i.e., $\mathbf{N}$ factors) of wake instabilities for diamond trip. Red and green curves correspond to two different mode families. (dashed line: $f=30 \mathrm{kHz}$, solid line: $f=40 \mathrm{kHz}$, dash-dot line: $f=50 \mathrm{kHz}$ ).

\section{Pressure Fluctuations Associated with Wake Instabilities}

The roughness configurations M6T and M6F were selected somewhat artificially to allow a meaningful comparison with the previously obtained results at Mach 3.5. Unlike the M3.5T case where both modes were deemed to have comparable significance for transition on the basis of their amplification factors, the odd modes have higher amplification potential in the M6T and M6F cases. These differences indicate that, despite the partial dynamic similarity among all three cases and the qualitatively similar mean velocity contours, the instability characteristics of the roughness element wake can be a sensitive function of the flow parameters. A somewhat analogous finding was noted in Ref 4 in the context of the Mach 3.5 case alone, where the even modes were found to rapidly become less important as the roughness height was reduced.

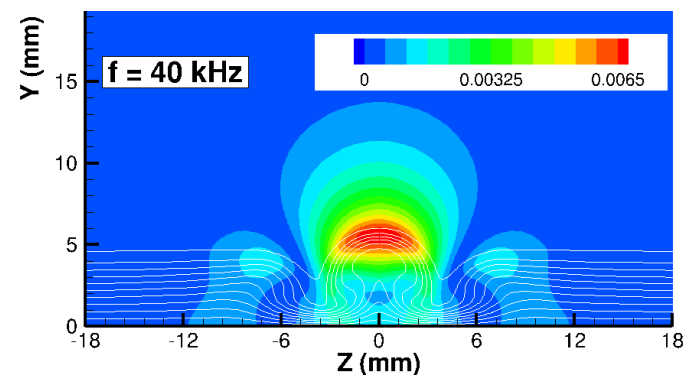

(a) Even mode $S(f=40 \mathrm{kHz})$.

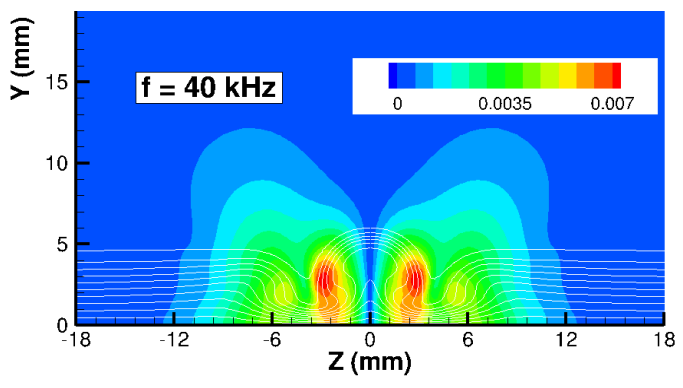

(b) Odd mode SI $(f=40 \mathrm{kHz})$.

Figure 13. Representative mode shapes of pressure fluctuations (non-dimensionalized with respect to $\left.\rho_{\infty} U_{\infty}^{2}\right)$ due to even and odd mode disturbances at $x=0.575 \mathrm{~m}\left(\left(x-x_{r}\right) / k \approx 92\right)$. (White lines indicate the associated contours of basic state $u$-velocity distribution.) Mode shapes are normalized such that the peak velocity fluctuation (scaled by free-stream velocity) has unit magnitude.

The M6T case illustrates the modal complexity of wake instabilities behind discrete roughness elements. The presence of even modes that are concentrated in regions of high spanwise gradients of the basic state (in contrast to the typical location of highest wall-normal gradients in the Mach 3.5 case) has been established for the first time. Of course, the roughness elements examined herein are shallow (with a spanwise width that is more than twice the boundary layer thickness and between 4 to 5 times the roughness height) in comparison with the cylindrical trip with a relatively small diameter used in the Mach 6 experiments at Purdue University. Future computations will focus on the latter case, particularly to examine how the reduced spanwise width of the wake in relation to the length scale of the second mode instabilities of the unperturbed boundary layer influences the characteristics of the wake instabilities. Given the availability of transition measurements for this case, the predicted amplification rates of the 
streak instabilities could be integrated in the streamwise direction to develop $\mathrm{N}$-factor correlations for the onset of transition or, at least, the expected trends in the transition location.

Due to the challenges in making detailed, off-surface measurements in high-speed boundary layers, the measurement of surface pressure fluctuations may provide a promising alternative to track the evolution of boundary layer instabilities. ${ }^{6}$ Representative mode shapes of pressure fluctuations associated with even and odd modes of instability at a representative location just behind the roughness element are plotted in Figs. 13(a) and 13(b), respectively. The peak surface pressure fluctuations along the surface were found to be weaker than the off-surface maxima, but by a modest factor of approximately 4.84 in the case of the even mode and an even smaller factor of approximately 1.22 in the case of the odd mode. Due to the antisymmetric behavior of the odd mode, a transducer at the symmetry plane that has a sufficiently small sensing area would respond only to the even mode fluctuations. More complex mode shapes are observed for other modal families (not shown). The different footprints of these modes along the surface indicate the need to have multiple pressure transducers across the spanwise width of the wake in order to obtain reliable measurements of the surface pressure fluctuations.

\section{Roughness Interaction with Instability Modes in Incoming Flow}

The streak instabilities are known to weaken considerably when the roughness height parameter is reduced. ${ }^{4}$ If the streak modes cannot achieve sufficiently large amplification factors, they cannot lead to transition on their own and, hence, an alternate explanation must be sought for the upstream movement in transition at the smaller (i.e., barely post-critical) roughness heights. It seems logical to approach this problem from the zero roughness limit, when the onset of transition would be caused by the instability modes of the unperturbed boundary layer flow, e.g., first mode instabilities in the M3.5T case. The effect of roughness induced mean flow modification on the growth of first mode perturbations is examined next.

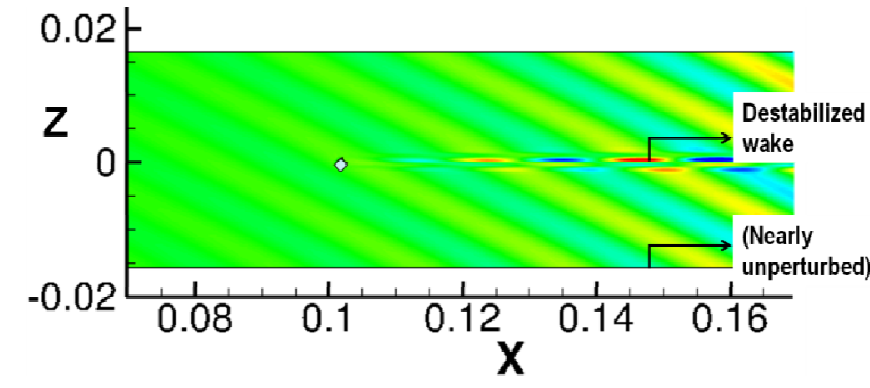

(a) Contours of instantaneous streamwise velocity fluctuation at fixed height above the plate.

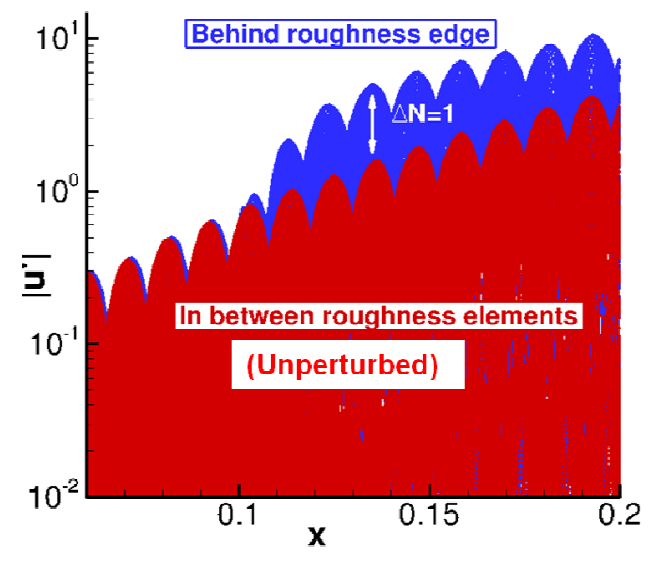

(b) Streamwise evolution of rms fluctuation at fixed height within two selected spanwise planes shown in part (a) (red: nearly unperturbed boundary layer away from roughness; blue: near the spanwise edge of the trip).

Figure 14. Interaction of first mode perturbations from incoming boundary layer with mean flow modified by roughness element.

Direct numerical simulations were performed to investigate whether the wake instability modes can be excited via roughness-induced scattering of an arbitrary disturbance field in the boundary layer flow ahead of the roughness element. The flow solver used for this DNS was the same as the previous study ${ }^{4}$ related to the receptivity and nonlinear breakdown of roughness induced streaks. To allow high-order computations with a body-fitted grid, the original diamond geometry of the baseline trip from Section 2 was replaced with a smooth version without any sharp corners. The smoothing of the diamond geometry, while essential for the numerical simulation, was not expected to impact the qualitative outcome pertaining to the interaction of upstream unsteadiness with the roughness element. 
The flow conditions were the same as the M3.5T case, but the trip location was moved downstream to allow the first mode wave to have a reasonably large growth rate at the trip location.

To investigate the interaction of first mode instabilities with the roughness induced mean flow perturbation, unsteadiness was introduced in the form of an oblique, time harmonic first mode wave forced through a localized surface actuator sufficiently ahead of the roughness element. The frequency of this time harmonic forcing was set equal to $30 \mathrm{kHz}$ and the spanwise wavelength was equal to $0.035 \mathrm{~m}$. Fig. 14(a) shows the visualization of the unsteady flow field at a fixed height above the plate, close to where the rms fluctuations in streamwise velocity achieve their peak levels. The streamwise evolution of rms velocity fluctuation within a pair of selected spanwise planes is shown in Fig. 14(b). Each red curve in Fig. 14(b) indicates the evolution of the velocity rms at a fixed distance from the surface, within a spanwise plane that is displaced from the center plane by approximately one half of the spanwise wavelength. Each blue curve corresponds to analogous variation within the spanwise plane that is approximately aligned with the edge of the roughness element. A comparison between the envelopes of the red and the blue curves indicates the enhanced growth of first mode perturbations within the roughness wake. The differential in $\mathrm{N}$-factor within the wake and away from the wake, respectively, corresponds to approximately $\Delta \mathrm{N}=$ 1.

The above results indicate that roughness induced mean flow perturbation can enhance the growth rates of first mode instabilities, contributing to a modest upstream movement in the transition onset location. However, additional computations must be performed to establish the generality of this finding, both with respect to a broader range of instability frequencies and spanwise wavenumbers, as well as smaller roughness heights.

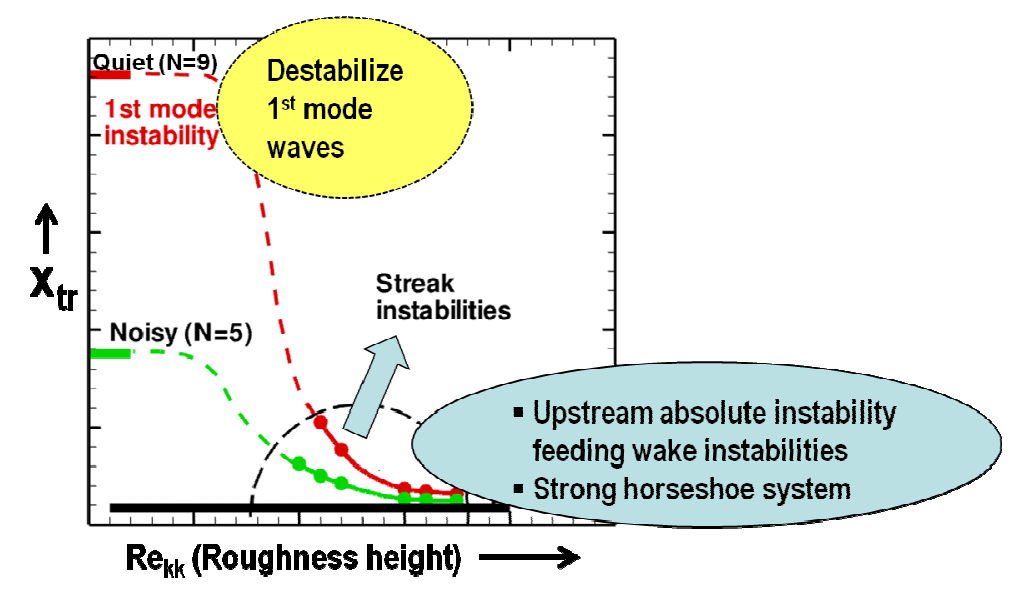

Fig. 15. Morphology of computationally inferred transition mechanisms for an isolated roughness element of varying height.

Fig. 15 presents a summary of the computationally inferred mechanisms for the transition due to a single isolated trip of varying height, based on a combination of computations from this paper and those presented in the previous work outlined in Section 1.

\section{Effects of Interaction Between Multiple Roughness Elements}

Naturally occurring surface nonuniformities, such as the roughness shapes induced by surface ablation, tend to be spatially distributed in nature. Thus, it is important to investigate the effects of spatial proximity between the individual roughness elements, particularly when certain elements lie within the wake of upstream elements. To study the interaction between multiple trips aligned with the flow direction in a simple context, the flow past a pair of isolated diamond trips (see Fig. 16) is examined next. The width of each trip is fixed at $b=1.27 \mathrm{~mm}$ while the streamwise spacing between the respective centers corresponds to $d=4.0 \mathrm{~mm}$. Figure 17 compares the streak amplitudes associated with the trips in tandem with those due to a single trip with the same height. Results for the relatively small height of $k=0.1 \mathrm{~mm}$ are shown in Fig. 17(a), whereas those for $k=0.25 \mathrm{~mm}$ are shown in Fig. 17(b). 


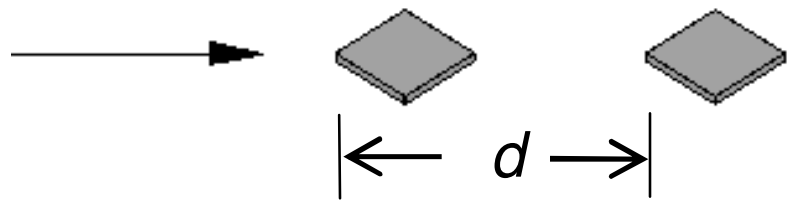

Figure 16. Schematic of a pair of trips in tandem arrangement (streamwise spacing between the centers of the two trips is given by $d=4.0 \mathrm{~mm}$ ).

The streak amplitudes produced by a single trip with $k=0.1 \mathrm{~mm}$ are weak enough that no significantly strong wake instability had been noted for that case. However, the flow approaching the downstream trip has sufficiently recovered from the relatively strong nearfield disturbance due to the upstream trip, that the second trip is able to produce a comparable wake disturbance augmenting the streak amplitudes due to the upstream trip. Thus, it is possible that, at the present combination of trip spacing and trip height, the introduction of additional trips would lead to sufficient reinforcement of the streak amplitude and, hence, to strong enough wake instabilities that could potentially cause transition even at a smaller roughness height. The augmentation of streak amplitude (also noted in additional computations with more than two roughness elements which are not included in this paper) is consistent with the large Reynolds number asymptotic theory by Goldstein et al. ${ }^{26}$ for sufficiently small roughness heights. However, the streak amplitudes at smaller heights decay more rapidly than those at larger roughness heights ${ }^{4}$ and this decay would limit how small the height of the distributed roughness can be while still producing a large enough impact on the transition process.

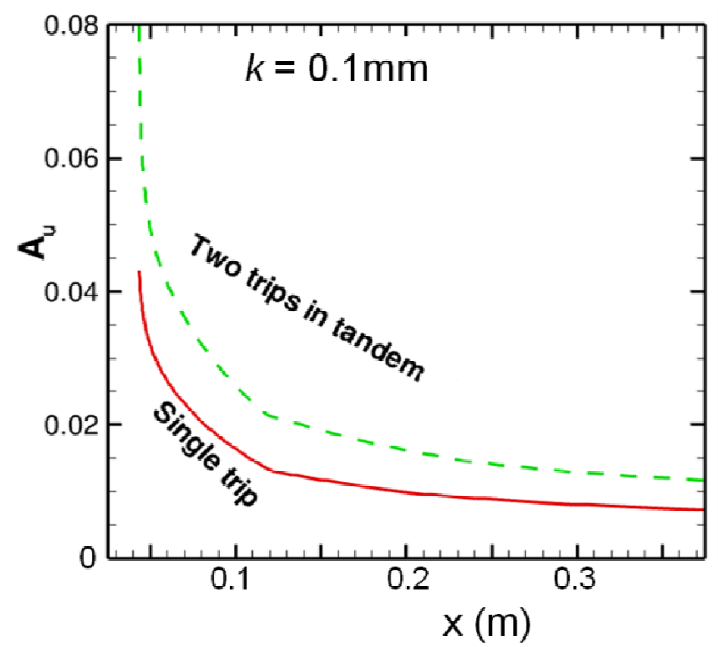

(a) Smaller trip height: $k=0.1 \mathrm{~mm}$.

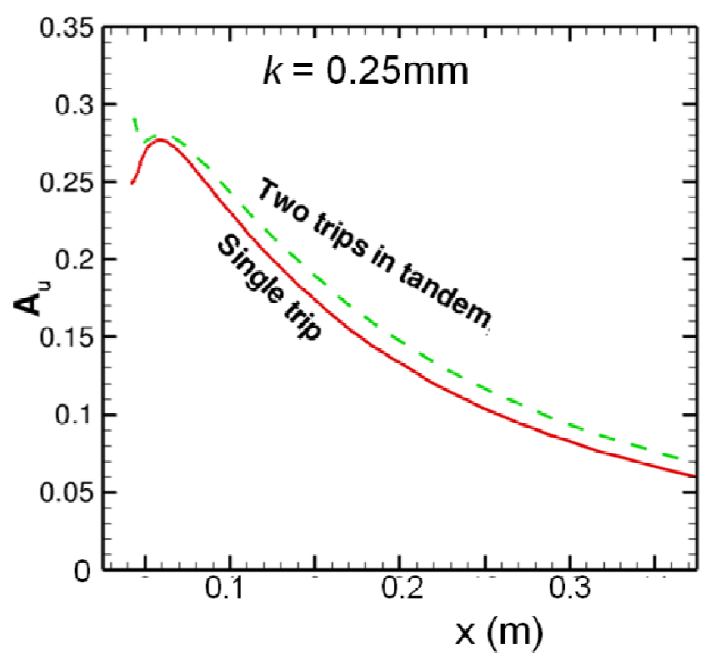

(b) Larger trip height: $k=0.25 \mathrm{~mm}$.

Figure 17. Comparison of streak amplitude evolution for a single trip and a pair of trips in tandem (streamwise spacing between the centers of the two trips is given by $d=4.0 \mathrm{~mm}$ ).

The larger trip height of $0.25 \mathrm{~mm}$ leads to wake instabilities with an $\mathrm{N}$-factor greater than 5 and, hence, has the potential to cause transition on its own in a noisy environment. ${ }^{4}$ Due to the stronger nearfield disturbance near the trip location (not shown in Fig. 17), the flow approaching the downstream trip with $k=0.25 \mathrm{~mm}$ has not recovered as much as that in the $k=0.1 \mathrm{~mm}$ case. This leads to a significantly weaker augmentation of the streak amplitude as seen from Fig. 17(b) and, hence, to a rather small upstream shift in transition location as a result of the downstream roughness element.

\section{Concluding Remarks}

This paper presented computational results for an isolated roughness element in zero pressure gradient boundary layers at Mach 3.5 and 5.9. The Mach 5.9 cases included flow conditions that are relevant to both ground facility experiments and high altitude flight ("cold wall" case). Regardless of the Mach number, the mean flow distortion 
due to the roughness element is characterized by long lived streamwise streaks in the roughness wake, which can support instability modes that did not exist in the absence of the roughness element. Instability computations for the Mach 5.9 cases reveal the presence of wake instability modes that are partly similar to those computed previously for a Mach 3.5 boundary layer with nearly same values of scaled roughness height $k / \delta$ and roughness height Reynolds number $R e_{k k}$, despite the underlying differences in the instability dynamics of the underlying boundary layer flows. The higher Mach number cases reveal a variety of mode shapes with velocity fluctuations concentrated in different localized regions of high base flow shear and the frequency range of the most unstable wake disturbances overlaps with the frequencies of first and second mode disturbances in the unperturbed flow. Some of the wake instability modes are concentrated near the top of the mushroom shaped structure in base flow velocity contours while the others exhibit most intense fluctuations on the side of the mushroom. At the roughness height of $k / \delta \approx 0.55$, the odd (i.e., antisymmetric) wake modes have higher $\mathrm{N}$-factor values than the even modes of instability in both Mach 6 cases.

Coordinated experimental measurements that capture sufficient details of the complex instability field would enable definitive verification of these mechanisms. It is shown that, in the higher Mach number cases (wherein the dominant modes of instability in the unperturbed boundary layer flow correspond to second mode disturbances), the disparity between the frequencies and growth rates of the wake instability modes and those of the instability modes outside of the wake region shrinks relative to the lower Mach number case where the outer modes correspond to oblique first mode instabilities with spanwise scales that are significantly larger than the boundary layer thickness.

This paper also presented initial results for a cylindrical roughness element that was employed during a recent experiment at Purdue University. This roughness element had a smaller planform size relative to both the diamond trip considered herein and to the local boundary layer thickness. While the mean wake flow in this case was seen to be qualitatively similar to that observed behind the diamond trip, additional computations are necessary to characterize the instability growth in the wake and compare it with the experimental measurements.

Preliminary computations for multiple roughness elements arranged in tandem indicate the potential for constructive reinforcement of streak amplitudes within the roughness wake, suggesting that the critical height for multiple roughness elements could be smaller than that for an isolated roughness element.

\section{ACKNOWLEDGMENT}

The work of NASA authors was performed as part of the Aerodynamics, Aerothermodynamics, and Plasma Dynamics (AAP) discipline of the Hypersonics Project of NASA's Fundamental Aeronautics Program (FAP). Development, validation, and improvements to the stability analysis code were carried out under the Supersonic Cruise Efficiency - Airframe component of the Supersonic Project. Assistance by Dr. Minwei Wu of NIA during a part of these computations is also acknowledged.

\section{REFERENCES}

${ }^{1}$ Van Driest, E. R. and McCauley, W. D., "The Effect of Controlled Three-Dimensional Roughness on Boundary-Layer Transition at Supersonic Speeds," J. Aero. Sc., Vol. 27, No. 4, pp. 261-271, 1960.

${ }^{2}$ Casper, K., Wheaton, B., Johnson, H., and Schneider, S., "Effect of Freestream Noise on Roughness-Induced Transition at Mach 6," AIAA Paper 2008-4291, 2008.

${ }^{3}$ Choudhari, M., Li, F., and Edwards, J. A., "Stability Analysis of Roughness Array Wake in a High-Speed Boundary Layer," NASA FAP/HYP/AAP Quarterly Newsletter, March 2008 (also published as AIAA Paper 2009-0170).

${ }^{4}$ Choudhari, M., Li, F., Wu, M., Chang, C.-L., and Edwards, J. A., "Laminar Turbulent Transition behind Discrete Roughness Elements in a High-Speed Boundary-Layer,” AIAA Paper 2010-1575, 2010.

${ }^{5}$ Chang, C., Choudhari, M., and Li, F., "Numerical Computations of Hypersonic Boundary-Layer over Surface Irregularities," AIAA Paper 2010-1572, 2010.

${ }^{6}$ Wheaton, B. and Schneider, S., "Roughness-Induced Instability in a Laminar Boundary Layer at Mach 6," AIAA Paper 2010-1574, 2010.

${ }^{7}$ Bartkowicz, M., Subbareddy, P. K., and Candler, G., "Numerical Simulations of Roughness Induced Instability in the Purdue Mach 6 Wind Tunnel," AIAA Paper 2010-4273, 2010.

${ }^{8}$ Klebanoff, P., Cleveland, W. G., and Tidstrom, K. D., "On the Evolution of a Turbulent Boundary Layer Induced by a Three-Dimensional Roughness Element,” J. Fluid Mech., Vol. 237, pp. 101-187, 1992.

${ }^{9}$ Choudhari, M. and Fischer, P., "Roughness-Induced Transient Growth,” AIAA Paper 2005-4765, 2005.

${ }^{10}$ Chang, C.-L., Choudhari, M., Venkatachari, B. S., and Li, F., "Effects of Cavities and Protuberances on Transition over Hypersonic Vehicles," AIAA Paper 2011-3245, 2011. 
${ }^{11}$ Danehy, P. M., Garcia, A. P., Borg, S., Dyakonov, A. A., Berry, S. A., (Wilkes)Inman, J. A., and Alderfer, D. W., "Fluorescence Visualization of Hypersonic Flow Past Triangular and Rectangular Boundary-Layer Trips," AIAA Paper 2007-536, 2007.

${ }^{12}$ Berry, S. A., Chen, F.-J., Wilder, M., and Reda, D., "Boundary Layer Transition Experiments in Support of the Hypersonics Program," AIAA Paper 2007-4266, 2007.

${ }^{13}$ Campbell, C. H., Garske, M., Kinder, J., and Berry, S. A., "Orbiter Entry Boundary Layer Flight Testing," AIAA Paper 2008-635, 2008.

${ }^{14}$ Horvath, T. J., Berry, S. A., Schwarz, R., Daryabeigi, K., Splinter, S., and Ross, M., "Assessment and Mission Planning Capability for Quantitative Aerothermodynamic Flight Measurements Using Remote Imaging," AIAA Paper 2008-4022, 2008.

${ }^{15}$ Marxen, O., Iaccarino, G., and Shaqfeh, E. S. G., "Numerical Simulation of the Effect of a Roughness Element on High-Speed Boundary-Layer Instability,” AIAA Paper 2008-4400, 2008.

${ }^{16}$ Greene, P., "A Numerical Study of Purdue's Mach 6 Tunnel with a Roughness Element," AIAA Paper 2009-0174, 2009.

${ }^{17}$ Redford, J. A., Sandham, N. D., and Roberts, G. T., "Roughness-Induced Transition of Compressible Laminar Boundary Layers", In Schlatter, Philipp and Henningson, Dan S. (eds.), Seventh IUTAM Symposium on LamierTurbulent Transition: Proceedings of the Seventh IUTAM Symposium on Laminar-Turbulent Transition, Stockholm, Sweden, pp. 337-342, 2009.

${ }^{18}$ Iyer, P. S., Muppidi, S., and Mahesh, K., "Transition of Hypersonic Flow past Flat Plate with Roughness Elements", AIAA Paper 2010-5015, 2010.

${ }^{19}$ Yoon, S., Barnhardt, M., and Sozer, E., "Hypersonic Transition to Turbulence Triggered by Isolated Roughness Elements," AIAA Paper 2011-565, 2011.

${ }^{20}$ Passaro, A and Baccarella, D., "Roughness Induced Transition in Hypersonic Regime Over a Flat Plate," AIAA Paper 2011-2375, 2011.

${ }^{21}$ Groskopf, G., Kloker, M. J., and Stephani, K. A., "Temperature/Rarefaction Effects in Hypersonic Boundary-Layer Flow with an Oblique Roughness Element," AIAA Paper 2011-3251, 2011.

${ }^{22}$ Reda, D. C., Wilder, M. C., and Prabhu, D. K., "Transition Experiments on Blunt Bodies with Isolated Roughness Elements in Hypersonic Free Flight," AIAA Paper 2010-1367, 2010.

${ }^{23}$ Berry, S. A., Chen, F.-J., Wilder, M., and Reda, D., "Boundary Layer Transition Experiments in Support of the Hypersonics Program," AIAA Paper 2007-4266, 2007.

${ }^{24} \mathrm{Li}$, F. and Choudhari, M., "Spatially Developing Secondary Instabilities and Attachment Line Instability in Supersonic Boundary Layers," AIAA Paper 2008-590, 2008.

${ }^{25} \mathrm{Li}$, F. and Choudhari, M., "Spatially Developing Secondary Instabilities in Compressible Swept Airfoil Boundary Layers," Theoretical and Computational Fluid Dynamics, Vol. 25, pp. 65-84, 2011.

${ }^{26}$ Goldstein, M. E., Sescu, A., Duck, P. W., and Choudhari, M., "The Long Range Persistence of Wakes behind a Row of Roughness Elements,” J. Fluid Mech., Vol. 644, pp. 123-163, 2010. 